\title{
Models for random Constraint Satisfaction Problems.
}

\author{
Michael Molloy \\ Dept. of Computer Science \\ University of Toronto \\ Toronto, ON, Canada \\ molloy@cs.toronto.edu
}

October 12,2000

\begin{abstract}
We introduce a class of models for random Constraint Satisfaction Problems. This class includes and generalizes many previously studied models. We characterize those models from our class which are asymptotically interesting in the sense that the limiting probability of satisfiability changes significantly as the number of constraints increases. We also discuss models which exhibit a sharp threshold for satisfiability in the sense that the limiting probability jumps from 0 to 1 suddenly as the number of constraints increases.
\end{abstract}

In this paper, we introduce a new general model for generating random Constraint Satisfaction Problems. We will generate problems on $n$ variables, where each variable has a domain of $d \geq 2$ permissable values, and each constraint is on $k$ variables for some fixed $k \geq 2$. For the sake of simplicity, we assume that each variable has the same domain, $\mathcal{D}=\left\{\delta_{1}, \ldots, \delta_{k}\right\}$, although this model can be easily modified to deal with more general situations. All asymptotics will be as $n \rightarrow \infty$, and so $d, k$ are considered to be constants. Thus, typically $n$ will be much larger than either $d$ or $k$.

This model generalizes many previously studied models, including virtually all well-studied models of Contraint Satisfaction Problems, random instances of $k$-SAT, and CSP's which are equivalent to determining whether a random graph is $k$-colourable. Using straightforward methods to simulate constraints of size $r$ by constraints of size $t$ for any $t>r$, this model also generalizes models with mixed constraint sizes, such as the $(2+p)$-SAT model studied in [4]. 
Given a $k$-tuple of variables, $\left(x_{1}, \ldots, x_{k}\right)$, a restriction on $\left(x_{1}, \ldots, x_{k}\right)$, is a $k$-tuple of values $R=\left(\delta_{1}, \ldots \delta_{k}\right)$ where each $d_{i} \in \mathcal{D}$. For each $k$-tuple $\left(x_{1}, \ldots, x_{k}\right)$, the set of restrictions on that $k$-tuple is called a constraint. The empty constraint is the constraint which contains no restrictions. We say that an assignment of values to the variables of a constraint $C$ satisfies $C$ if that assignment is not one of the restrictions in $C$. An assignment of values to all variables in a CSP satisfies that CSP if every constraint is simultaneously satisfied. The constraint hypergraph of a CSP is the $k$-uniform hypergraph whose vertices correspond to the variables, and whose hyperedges correspond to the $k$-tuples of variables which have constraints.

In studying large random CSP's one of the most natural questions is to determine the liklihood of the problem being satisfiable. For example, we might show that for some settings of the parameters in the model, a random CSP is almost surely ${ }^{1}$ (a.s.) satisfiable, while for other settings it is a.s. non-satisfiable. Of particular interest, are situations where we have a threshold behaviour, such as a situation where as we modify the number of constraints, a random CSP moves from being a.s. satisfiable to a.s. not satisfiable. Besides being theoretically interesting, such models are useful for practical purposes, since it is believed that CSP's selected from such a model with the number of constaints very close to the threshold, are very difficult to solve and so provide good test problems (see [8] for some of the first empirical evidence of this hypothesis).

Some natural and important models are known to have a threshold behaviour, most notably, random instances of $k$-SAT. This fact is probably what led others to assume that the same is true of many models of random CSP. Unfortunately, and somewhat surprisingly, such assumptions turned out to be often invalid, as discussed below. Thus, a mathematically rigorous examination of the threshold behaviour of models of random CSP is neccessary. Such a study is the goal of the present paper.

Until recently, the most commonly used random CSP models were the following:

Model A1: Specify $M, p$ (typically $M=c n$ for some constant $c$ and $0<p \leq 1$ is independent of $n$ ). First choose a random $k$-uniform hypergraph on $n$ vertices with $M$ hyperedges (where each such hypergraph is equally likely), which will be the constraint hypergraph of our problem. Next, for each hyperedge $e$, we choose a constraint on the $k$ variables of $e$ as follows: Each of the $d^{k}$ possible restrictions is chosen to be present with probability $p$ (where each choice is, of course, independent of the corresponding choices for other potential contraints).

Model A2: Specify $M, m$ (typically $M=c n$ for some constant $c$ and

\footnotetext{
${ }^{1}$ A random CSP almost surely satisfies property $\mathrm{P}$ if $\lim _{n \rightarrow \infty} \operatorname{Pr}(P)=1$.
} 
$0<p \leq 1$ is independent of $n$ ). Choose the constraint hypergraph as in Model A1. For each hyperedge $e$, we choose a constraint with exactly $m$ restrictions uniformly at random from the set of $\left(\begin{array}{c}d^{k} \\ m\end{array}\right)$ such constraints.

Remark 1: Alternatively, we could have chosen the constraint hypergraphs by making an independent choice for each potential hyperedge, deciding to put it in the hypergraph with probability $p=\frac{c \times k !}{n^{k-1}}$. This variation produces models which are equivalent to Models A1 and A2 in the sense that the models will be a.s. satisfiable and a.s. unsatisfiable for the same values of $c$. A similar remark applies to the other two models described below.

In [5], we observed that Model A1 is asymptotically uninteresting in the sense that as long as $M$ grows with $n$, the random CSP will be a.s. unsatisfiable. The problem is quite simple: a.s. there will be at least one constraint which is overconstraining in that there is no assignment to its variables which satisfies even that constraint, i.e. a constraint which includes every possible restriction amongst its $k$ variables. Model A2 avoided this particular problem, but had other equally damaging problems as long as $M$ was of order $\Theta(n)$ (as is usually the case) for all but small values of $m: m<d^{k-1}$ (Gent et al [11] showed that these small values of $m$ are indeed not flawed). [11] provides a survey of models used in previous research, and found that roughly $3 / 4$ of papers used models which were uninteresting in the sense described above. Furthermore, those models which were interesting (mostly Model A2 with $m<d^{k-1}$ ) seem to have been "accidentally" interesting in the sense that the authors were unaware that the small value they chose for $m$ was crucial.

So we proposed an alternative model:

Model B: Specify $M$ (typically $M=c n$ for some constant $c$ ). Choose a random CSP containing $M$ restrictions, where each such CSP is equally likely to be chosen.

We showed that this model was asymptotically interesting in the sense that for small values of $c$ a random CSP is a.s. satisfiable, while for large values of $c$ it is a.s. unsatisfiable.

Nevertheless, this model is rather unsatisfactory in the sense that the CSP's which it generates do not resemble CSP's that researchers tend to find interesting. In particular, almost every constraint contains only one restriction, while in typical CSP's most constraints tend to have several restrictions.

Here, we propose a model which is both asymptotically interesting, and which overcomes the main disadvantage of Model $B$, in that it can generate CSP's whose constraints have several restrictions. We aim to keep the model as simple as possible, while at the same time meeting these two (somewhat conflicting) goals. In particular, our model is symmetric in the variables. It is not neccessarily symmetric amongst the values of $\mathcal{D}$, although the parameters 
of the model can be set so that it is.

Gent et. al. [11] proposed another model which is both asymptotically interesting and which generates reasonably complex constraints. Their model turns out to be a special case of the model introduced here. In the terminology introduced subsequently in this paper, they proved that their model is very well behaved, en route to showing that it exhibits a transition.

Our new model is similar in flavour to Models A1 and A2, but we must take some care in the way that we choose the constraints in order to avoid overconstrained constraints and similar problems. In particular, we cannot just independently choose whether or not to put each potential restriction in the constraint - we must choose the entire constraint en masse.

So, we consider a canonical set of variables, $X_{1}, \ldots, X_{k}$, and the set of $2^{d^{k}}-1$ potential non-empty constraints on $X_{1}, \ldots, X_{k}$. We specify a probability distribution $\mathcal{P}$ which selects a single random constraint. For simplicity, we assume that $\mathcal{P}$ is independent of $n$.

New Model: Specify $M, \mathcal{P}$ (typically $M=c n$ for some constant $c$ ). First choose a random constraint hypergraph with $M$ hyperedges, in the usual manner. Next, for each hyperedge $e$, we choose a constraint on the $k$ variables of $e$ as follows: we take a random permutation from the $k$ variables onto $\left\{X_{1}, \ldots, X_{k}\right\}$ and then we select a random constraint according to $\mathcal{P}$, mapping it onto a constraint on our $k$ variables in the obvious manner.

Remark 2: Again, we could have chosen the constraint hypergraph by making an independent choice for each potential hyperedge, deciding to put it in the hypergraph with probability $p=\frac{c \times k !}{n^{k-1}}$. This variation produces a model which is, for our purposes, equivalent to the model as described above.

We set $\mathcal{C}=\operatorname{supp}(\mathcal{P})$, i.e. the set of constraints, $C$, for which $\mathcal{P}(C)>0$. It turns out, that whether or not this model is asymptotically interesting depends only on $\mathcal{C}$. In what follows, we will specify the exact conditions that $\mathcal{C}$ must satisfy in order for the model to be asymptotically interesting. To do so, we need a few definitions:

We use $C S P_{n, M}(\mathcal{P})$, to denote a random CSP drawn from our New Model with parameters $n, M, \mathcal{P}$. We occsionally omit the subscript $n, M$, depending on the context. We say that $\operatorname{CSP}(\mathcal{P})$ exhibits a partial transition if there exists constants $c_{1}, c_{2}>0$ such that

(i) if $M<c_{1} n$ then $C S P_{n, M}(\mathcal{P})$ is not a.s. unsatisfiable;

(ii) if $M>c_{2} n$, then $C S P_{n, M}(\mathcal{P})$ is a.s. unsatisfiable.

We say that $\operatorname{CSP}(\mathcal{P})$ exhibits a transition if there exists $c_{1}, c_{2}>0$ such that 
(i) if $M<c_{1} n$ then $C S P_{n, M}(\mathcal{P})$ is a.s. satisfiable;

(ii) if $M>c_{2} n$ then $C S P_{n, M}(\mathcal{P})$ is a.s. unsatisfiable.

Note that if $\operatorname{CSP}(\mathcal{P})$ exhibits a transition then it exhibits a partial transition.

Remark: It turns out that there is no choice of $C S P(\mathcal{P})$ and $c_{2}$ such that for all $M>c_{2} n, C S P_{n, M}(\mathcal{P})$ is neither a.s. satisfiable nor a.s. unsatisfiable. Thus there is no need for the obvious 3rd and 4th notions of "transition".

In the next two sections, we will characterize those models $C S P(\mathcal{P})$ which have transitions and partial transitions. We will see in that for each $\mathcal{C}$, we have that $C S P(\mathcal{P})$ exhibits a transition (partial transition) for at least one choice of $\mathcal{P}$ with $\mathcal{C}=\operatorname{supp}(\mathcal{P})$ iff $C S P(\mathcal{P})$ exhibits a transition (partial transition) for every such choice of $\mathcal{P}$. Thus, it makes sense to say that $\mathcal{C}$ exhibits a transition or a partial transition.

In the fourth section, we will discuss models which have a very sharp threshold for satisfiability.

A constraint $C$ is unsatisfiable if it has no satisfying assignments, i.e. if it contains every possible restriction. A constraint $C$ forbids $X_{i}=\delta$ if it has no satisfying assignments for which $X_{i}=\delta . X_{i}=\delta$ implies $X_{j}=\gamma$ in $C$ if there are no satisfying assignments for $C$ in which $X_{i}=\delta$ and $X_{j} \neq \gamma$. A constraint $C$ implies a property $P$ if every satisfying truth assignment for $C$ meets $P$.

An automorphism of $\mathcal{C}$ is a bijection $\phi: \mathcal{D} \rightarrow \mathcal{D}$ such that $C=\left\{\left(\delta_{1}^{1}, \ldots \delta_{k}^{1}\right), \ldots,\left(\delta_{1}^{t}, \ldots, \delta_{k}^{t}\right)\right\} \in$ $\mathcal{C}$ iff $\phi(C)=\left\{\left(\phi\left(\delta_{1}^{1}\right), \ldots, \phi\left(\delta_{k}^{1}\right)\right), \ldots,\left(\phi\left(\delta_{1}^{t}\right), \ldots, \phi\left(\delta_{k}^{t}\right)\right)\right\} \in \mathcal{C}$. We say that $\mathcal{C}$ is symmetric with respect to $\mathcal{D}$ if it is symmetric in in the following sense: For any two values $\delta, \gamma \in \mathcal{D}$, there is an automorphism $\phi$ of $\mathcal{C}$ such that $\phi(\delta)=\gamma$.

Note that we do not require the symmetry to extend to $\mathcal{P}$, i.e. it is possible that $\mathcal{P}(C) \neq \mathcal{P}(\phi(C))$. Furthermore, $\mathcal{C}$ does not need to be symmetric in $X_{1}, \ldots, X_{k}$. For example, it could be that every constraint $C \in \mathcal{C}$ implies that $X_{1}=X_{2} \neq X_{3}$, while at the same time $\mathcal{C}$ is symmetric with respect to $\mathcal{D}$. However, the fact that our model takes a random mapping of the variables from an edge of the constraint hypergraph onto $\left(X_{1}, \ldots, X_{k}\right)$ implies that the model is symmetric with respect to the variables.

Our new model generalizes several previously studied models of CSP and other problems which reduce to being random instances of CSP. For example, Model A1 is simply this model where $\mathcal{P}(C)=p^{|C|}(1-p)^{d^{k}-|C|}$. Model A2 is the case where $\mathcal{P}(C)=\left(\begin{array}{l}d^{k} \\ m\end{array}\right)^{-1}$ if $|C|=m$ and $\mathcal{P}(C)=0$ otherwise. Model $\mathrm{B}$, is virtually equivalent to the case where $\mathcal{P}(C)=\frac{1}{d^{k}}$ if $|C|=1$, and $\mathcal{P}(C)=0$ otherwise. The well-studied problem of whether a random instance of $k$-SAT is satisfiable is equivalent to Model B where $d=2$. The problem of deciding 
whether a random graph is $c$-colourable is equivalent to the case where $k=$ $2, d=c$ and $\mathcal{P}(C)=1$ where $C=\left\{\left(\delta_{1}, \delta_{1}\right), \ldots,\left(\delta_{c}, \delta_{c}\right)\right\}$. In each of these examples, $\mathcal{C}$ is symmetric. It is already known that the first of these examples does not exhibit even a partial transition, that the second one only exhibits a partial transition if $m<d^{k-1}$, the third exhibits a transition, and the other two exhibit transitions for $k, c>2$ and partial transitions for $k, c=2$.

In the final section of this paper, we will discuss reasonable ways to generate instances of CSP according to $C S P(\mathcal{P})$ for some distributions of $\mathcal{P}$ which have transitions or partial transitions, for experimental purposes.

It is important to note that by the nature of the asymptotics involved, this study is only relevant as the number of variables grows very high. So these asymptotic results might be completely out-of-sync with experimental results which, due to technological limitations, are usually performed on a small number of variables, often less than 50. In fact, it is precisely this difference between the behaviour of the model on small instances and its asymptotic behaviour which led to earlier false assumptions about the asymptotic behaviour of Models A1 and A2.

\section{The Symmetric Case}

In this section, we focus on the case where $\mathcal{C}$ is symmetric. We say that a symmetric $\mathcal{C}$ is well-behaved if it satisfies the following two properties:

1. There is no $C \in \mathcal{C}, \delta \in \mathcal{D}$ and canonical variable $X_{i}$ such that $C$ forbids $X_{i}=\delta$.

2. For every $\delta \in \mathcal{D}$, there is at least one $C \in \mathcal{C}$ such that $X_{1}=\delta, X_{2}=$ $\delta, \ldots, X_{k}=\delta$ does not satisfy $C$.

A symmetric $\mathcal{C}$ is very well-behaved if, in addition to properties 1,2 , it also satisfies:

3. If a CSP is formed using constraints from $\mathcal{C}$, and its constraint hypergraph is a cycle, then it must be satisfiable.

It is worth noting that if Property 1 holds, then there is no unsatisfiable constraint $C \in \mathcal{C}$, and so we avoid the main problem with Model A1. However, as we will see, for $\operatorname{CSP}(\mathcal{P})$ to not be a.s. unsatisfiable, it does not suffice

to merely have no unsatisfiable constraint $C \in \mathcal{C}$ - we need the full power of Property 1 in order to avoid a more subtle problem. 
Theorem 1 If $\mathcal{C}$ is well-behaved, then for every distribution $\mathcal{P}$ with $\mathcal{C}=\operatorname{supp}(\mathcal{P})$, $\operatorname{CSP}(\mathcal{P})$ exhibits a partial transition. If $\mathcal{C}$ is not well-behaved, then for every distribution $\mathcal{P}$ with $\mathcal{C}=\operatorname{supp}(\mathcal{P}), C S P(\mathcal{P})$ does not exhibit a partial transition.

Note that Theorem 1 immediately implies that Model A2 exhibits a partial transition iff $1 \leq m<k^{d-1}$.

Theorem 2 If $\mathcal{C}$ is very well-behaved, then for every possible distribution $\mathcal{P}$, $\operatorname{CSP}(\mathcal{P})$ exhibits a transition. If $\mathcal{C}$ is not very well-behaved, then for every possible distribution $\mathcal{P}, \operatorname{CSP}(\mathcal{P})$ does not exhibit a transition.

Proof of Theorem 1: First we prove that our two properties are neccessary for $C S P(\mathcal{P})$ to have a partial transition.

Suppose that Property 2 does not hold. Then for at least one value $\delta$, setting each $X_{i}=\delta$ will satisfy any $C \in \mathcal{C}$, and so setting every variable in our CSP equal to $\delta$ will satisfy our formula with probability 1 , regardless of $M$ (in fact, since $\mathcal{C}$ is symmetric in $\mathcal{D}$, this is true for every $\delta \in \mathcal{D}$ ). Thus, there is no value of $M$ for which $C S P_{n, M}(\mathcal{P})$ is a.s. unsatisfiable.

Suppose that Property 1 does not hold, i.e. that there is some value $\delta \in \mathcal{D}$, canonical variable $X_{i}$, and $C \in \mathcal{C}$, such that $C$ forbids $X_{i}=\delta$. Since $\mathcal{C}$ is symmetric in $\mathcal{D}$, we have that for every $\delta \in \mathcal{D}$ there is some $C(\delta) \in \mathcal{C}$ such that $C$ forbids $X_{i}=\delta$.

We say that a variable $x$ is impossible if $x$ lies in at least $d$ hyperedges of our constraint hypergraph, $e_{1}^{j}, \ldots, e_{d}^{j}$, and each $e_{\ell}^{j}$ has the constraint $C\left(\delta_{\ell}\right)$ where $x$ is mapped onto $X_{i}$.

It is not hard to see that if $x$ is impossible then our formula is not satisfiable, since $x$ is forbidden from being assigned any of $\delta_{1}, \ldots, \delta_{d}$.

Suppose that $M=c n$ for any value of $c$.

Claim: A.s. $C S P_{n, M}(\mathcal{P})$ has an impossible variable.

Proof: First choose the constraint hypergraph. It is well known ${ }^{2}$ (and an easy application of Chebychev's Inequality) that a.s. there will be at least $\zeta n$ vertices with degree exactly $d$, such that no vertex lies in a 2 -cycle, for a particular constant $\zeta=\zeta(c, k, d)>0$. Let $A_{1}$ be the event that we do have this many such vertices. If $A_{1}$ holds then a simple greedy algorithm will produce an independent set $I$ of size at least $\frac{\zeta}{(k-1) d+1} n$ containing only vertices of degree $d$ : Simply add vertices of degree $d$ to $I$ one at a time. Each time we add a vertex to $I$, we forbid any of its $d(k-1)$ neighbours from being added to $I$. We will always be able to continue until $|I|>\frac{\zeta}{(k-1) d+1} n$.

\footnotetext{
${ }^{2}$ See $[7]$ for a proof of the case $k=2$. The proof for higher values of $k$ is similar.
} 
Next we choose the constraints for each hyperedge. Each vertex $v \in I$ will become impossible with some positive probability $p_{1}>0$, since $v$ does not lie in a 2-cycle. Furthermore, each such event is independent of the corresponding events for the other vertices in $I$, since $I$ is an independent set. Therefore, the probability that there is no impossible variable is at most $\operatorname{Pr}\left(\overline{A_{1}}\right)+(1-$ $p)^{\frac{\zeta}{(k-1) d+1} n}=o(1)$.

Thus, we have shown that if $\mathcal{C}$ is not well-behaved then either $C S P_{n, M}(\mathcal{P})$ is always a.s. unsatisfiable or it is always a.s. satisfiable, regardless of $\mathcal{P}$ and so $\mathcal{C}$ does not exhibit a partial transition.

Now we show that if $\mathcal{C}$ is well-behaved then it exhibits a partial transition for any choice of $\mathcal{P}$. First, we prove that Property 1 implies that we can take $c_{1}=\frac{1}{k(k-1)}$ :

It is well known ${ }^{3}$ that for $c<\frac{1}{k(k-1)}$, the probability that our constraint hypergraph is acyclic tends to a constant $\rho(c)>0$. It is not hard to show that if our constraint hypergraph is acyclic then Property 1 implies that our CSP is satisfiable. Thus the probability of satisfiability is at least $\rho$, and so we are not a.s. unsatisfiable.

For each component of the constraint hypergraph, we pick a variable to be the root, and set it arbitrarily. We then process each hyperedge in sequence, i.e. assign values to the variables of that hyperedge, such that every time we process a hyperedge, exactly one of its variables has already been set. Since no $C \in \mathcal{C}$ forbids any values from any variables, we will be able to find an assignment for the other variables on that hyperedge without violating the corresponding constraint.

Finally, we complete our proof by showing that Property 2 implies the existence of $c_{2}$, using what is by now a standard first moment argument.

Let $A$ be the number of satisfying assignments of our random CSP. We will prove that there exists $c_{2}$ such that if $p=c / n$ where $c>c_{2}$, then $\operatorname{Exp}(A)=o(1)$. This implies that a.s. $A=0$, i.e. our CSP is unsatisfiable.

There are $d^{n}$ truth assignments. Consider any one particular assignment $\mathcal{A}$. There is a value $\delta$ such that some set $S$ of at least $\frac{n}{d}$ variables are assigned $\delta$ in $\mathcal{A}$. By property 2, there is some constraint $C^{*} \in \mathcal{C}$ such that setting each $X_{i}=\delta$ violates $C^{*}$. The probability that $\mathcal{A}$ is a satisfying assignment is at most the probability that none of the $k$-tuples of $S$ is constrained by $C^{*}$. The probability that a randomly chosen constraint is $C^{*}$ applied to a $k$-tuple of $S$,

\footnotetext{
${ }^{3}$ See $[7]$ for a proof of the case $k=2$. See also the branching argument inspired by Karp [12] in [6]. The proof for higher values of $k$ is similar.
} 
is

$$
\mathcal{P}\left(C^{*}\right) \times\left(\begin{array}{c}
n / d \\
k
\end{array}\right) /\left(\begin{array}{l}
n \\
k
\end{array}\right) \approx \frac{\mathcal{P}\left(C^{*}\right)}{d^{k}} .
$$

From this fact, standard easy arguments yield that

$$
\begin{aligned}
\operatorname{Exp}(A) & \leq d^{n}\left(1-\frac{\mathcal{P}\left(C^{*}\right)}{d^{k}}\right)^{c n} \\
& <\mathrm{e}^{n \times\left(\ln d-\frac{c \mathcal{P}\left(C^{*}\right)}{d^{k}}\right)} \\
& =o(1)
\end{aligned}
$$

for $c>c_{2}=\frac{d^{k} \ln d}{\mathcal{P}\left(C^{*}\right)}$.

Remark: In fact, note that we have shown that our CNF is always a.s. unsatisfiable iff Property 1 does not hold, and it is always a.s. satisfiable iff Property 2 does not hold.

Proof of Theorem 2: First we show why property 3 is neccessary for our model to have a transition. Suppose that it doesn't hold, i.e. that there is some unsatisfiable CNF formula $C$ consisting of constraints from $\mathcal{C}$ and whose constraint hypergraph forms a $\ell$-cycle.

For any value of $c>0$, it is well known ${ }^{4}$ that the probability that a random hypergraph with $c n$ edges has an $\ell$-cycle tends to a constant $\zeta_{1}=\zeta_{1}(c, \ell)>0$. If our constraint hypergraph has an $\ell$-cycle, then the probability of that $\ell$ cycle being constrained by $C$ is equal to some constant $\zeta_{2}>0$. Therefore, the probability of being unsatisfiable tends to at least $\zeta_{1} \zeta_{2}>0$ and so we are not a.s. satisfiable for any value of $c$.

On the other hand, if property 3 does hold, then for $c<\frac{1}{k(k-1)}$ our CNF is a.s. satisfiable. It is well-knon that a.s. the constraint hypergraph will have no component with more than 1 cycle. If this happens, then the variables of each tree component can be set as in the proof of Theorem 1. The variables of each unicyclic component can be set as follows: first set the variables of the cycle in a satisfactory manner (which will always be possible by Property 3 ), and then set the remaining variables in the same manner as in a tree component.

The remainder of the theorem follows as in the proof of Theorem 1 .

In our proof, we showed that if $\mathcal{C}=\operatorname{supp}(\mathcal{P})$ exhibits a transition then $C S P(\mathcal{P})$ is a.s. satisfiable when $M$ is below the threshold for the constraint hypergraph to have a giant component. Not surprisingly, for many choices of $\mathcal{P}$, the same is still true for higher values of $M$. For example:

\footnotetext{
${ }^{4}$ See $[7]$ for a proof of the case $k=2$. The proof for higher values of $k$ is similar.
} 
Theorem 3 Suppose that $\mathcal{C}$ is such that for every minimally unsatisfiable formula $F$ whose constraints are drawn from $C$, the ratio of constraints to variables of $F$ is at least $\frac{1}{k-1}(1+\epsilon)$ for some constant $\epsilon>0$. Then there is a constant $\delta>0$ such that for any $\mathcal{P}$ with $\operatorname{supp}(\mathcal{P})=\mathcal{C}, C S P(\mathcal{P})$ is a.s. satisfiable for $M \leq \frac{1}{k(k-1)}(1+\delta) n$.

Note that for the hypothesis of Theorem 3 to hold, $\mathcal{C}$ must be very wellbehaved since a cycle has a hyperedge/vertex ratio of exactly $\frac{1}{k-1}$, and a tree has an even smaller ratio.

Proof This is a standard type of argument in random graph theory, but we include it here because it is not well known.

We can assume that $\epsilon$ is arbitrarily small, say $\epsilon \leq \frac{1}{2}$. Suppose that $M=c n$ where $c=\frac{1}{k(k-1)}(1+\delta)$, and $\delta<\epsilon$ is a small constant to be specified later. We will show that the expected number of subgraphs of our random constraint hypergraph which have the required hyperedge/vertex ratio is $o(1)$. Thus, a.s. none exist and so the CSP must be satisfiable since any unsatisfiable formula contains a minimally unsatisfiable subformula.

We can assume that such a subgraph has no tree or unicyclic components, since deleting such a component will only increase the hyperedge/vertex ratio. Thus, the subgraph must lie within the giant component. The size of the giant component is well-studied ${ }^{5}$ and is a.s. at most $\alpha n$ where $\alpha$ tends to zero with $\delta$. Furthermore Thus, we can restrict our attention to subgraphs of size at most $\alpha n$.

For any $0<a \leq \alpha n$, we consider the expected number of subgraphs on $a$ vertices with $b=\frac{1}{k-1}(1+\epsilon) a$ edges. There are $\left(\begin{array}{l}a \\ 2\end{array}\right)$ choices for the vertices, and $\left(\begin{array}{c}M \\ b\end{array}\right)$ choices for the edges. Given such choices, the probability that all the $b$ edges lie within the $a$ vertices is at most $\left(\frac{a}{n}\right)^{k b}$. Therefore, the expected number of such subgraphs is at most:

$$
\begin{aligned}
\left(\begin{array}{l}
n \\
a
\end{array}\right)\left(\begin{array}{c}
M \\
b
\end{array}\right)\left(\frac{a}{n}\right)^{k b} & \leq\left(\frac{\mathrm{e} n}{a}\right)^{a}\left(\frac{\mathrm{e} M}{b}\right)^{b}\left(\frac{a}{n}\right)^{k b} \\
& =\left(\frac{\mathrm{e}^{1+(1+\epsilon) /(k-1)} c^{(1+\epsilon) /(k-1)}(k-1)}{1+\epsilon}\left(\frac{a}{n}\right)^{\epsilon}\right)^{a} \\
& =\left(K\left(\frac{a}{n}\right)^{\epsilon}\right)^{a},
\end{aligned}
$$

where $K=K(\epsilon)$ is a constant. For $a \leq n^{\epsilon / 4}$, this expression is $\left(\Theta\left(n^{-\epsilon(1-\epsilon / 4)}\right)\right)^{a}<$ $n^{-\epsilon / 2}$. And for $a<\alpha n$, it is less than $\left(\frac{1}{2}\right)^{a}$ where $\alpha$ is sufficiently small in terms

\footnotetext{
${ }^{5}$ See, for example, $[7]$ for the case $k=2$. The case $k>2$ is amenable to similar analysis, or to the simpler branching process analysis found in [6] and inspired by [12].
} 
of $\epsilon$. Thus the expected number of subgraphs is less than

$$
\sum_{a=1}^{n^{\epsilon / 4}} n^{-\epsilon / 2}+\sum_{a \geq n^{\epsilon / 4}}\left(\frac{1}{2}\right)^{a}=o(1),
$$

for $\delta=\delta(\alpha)$ sufficiently small.

Theorem 3 applies to several common models. For example, a minimally non- $r$-colourable graph has edge/vertex ratio at least $r / 2$ since such a graph has minimum degree at least $r$. Also, a minimally unsatisfiable instance of $k$-SAT has clause/variable ratio at least $2 / k$ since it cannot contain any pure literals. It would be interesting to characterize the distributions $\mathcal{P}$ for which $C S P(\mathcal{P})$ is a.s. unsatisfiable with $M=c n$ for all $c>\frac{1}{k(k-1)}$.

\section{The asymmetric case}

Here we extend the definitions and theorems of the previous section, to the case where $\mathcal{C}$ is not neccessarily symmetric in $\mathcal{D}$.

We say that a value $\delta$ is 0 -bad, if there is some canonical variable $X_{i}$, and constraint $C \in \mathcal{C}$ such that $C$ forbids $X_{i}=\delta$. We say that $\delta$ is $j$-bad if there is some canonical variable $X_{i}$, and constraint $C \in \mathcal{C}$ such that $C$ implies that if $X_{i}=\delta$ then at least one other canonical variable must be assigned a $j^{\prime}$-bad value for some $j^{\prime}<j$. A value is bad if it is $j$-bad for some $j$. A value is good if it is not bad.

If $\mathcal{C}$ is symmetric then, of course, if one value is bad then all values are bad, and so the existence of a bad value would violate Property 1 from the previous section. However, to allow for the case that $\mathcal{C}$ is asymmetric, we must modify Property 1 . We say that $\mathcal{C}$ is well-behaved if it satisfies the following two properties:

1'. There is at least one good value in $\mathcal{D}$.

2. For every $\delta \in \mathcal{D}$, there is at least one $C \in \mathcal{C}$ such that $X_{1}=\delta, X_{2}=$ $\delta, \ldots, X_{k}=\delta$ does not satisfy $C$.

Remark: It is an easy exercise to show that Properties 1' and 2 imply that there are at least 2 good values in $\mathcal{D}$.

We also must modify Property 3 slightly. We say that $\mathcal{D}$ is very well-behaved if, in addition to properties 1 ',2, it also satisfies: 
3'. If a CSP is formed using constraints from $\mathcal{C}$, and its constraint hypergraph is a cycle, then it must have a satisfying assignment where no variable is assigned a bad value.

We have the analagous results from the previous section:

Theorem 4 If $\mathcal{C}$ is well-behaved, then for every possible distribution $\mathcal{P}, C S P(\mathcal{P})$ exhibits a partial transition. If $\mathcal{C}$ is not well-behaved, then for every possible distribution $\mathcal{P}, \operatorname{CSP}(\mathcal{P})$ does not exhibit a partial transition.

Theorem 5 If $\mathcal{C}$ is very well-behaved, then for every possible distribution $\mathcal{P}$, $\operatorname{CSP}(\mathcal{P})$ exhibits a transition. If $\mathcal{C}$ is not very well-behaved, then for every possible distribution $\mathcal{P}, \operatorname{CSP}(\mathcal{P})$ does not exhibit a transition.

The proofs are along the same lines as those in the previous section. We present only the portions of the proofs which are different.

Proof of Theorem 4: First we show that if condition 1' does not hold, then for every distribution $\mathcal{P}$ and value $c>0, C S P(\mathcal{P})$ is a.s. unsatisfiable.

For each $j \geq 0$ we let $b_{j}$ denote the number of $j$-bad values. Thus $\sum_{j>0} b_{j}=$ $d$. For each 0 -bad value $\delta$, we let $C(\delta)$ be the constraint and $X_{i(\delta)}$ be the canonical variable such that $C(\delta)$ forbids $X_{i(\delta)}=\delta$. Similarly, if $j \geq 1$ then for each $j$-bad value $\delta$, we let $C(\delta)$ be the constraint and $X_{i(\delta)}$ be the canonical variable such that if $X_{i(\delta)}=\delta$ then at least one other canonical variable must take a $j^{\prime}$-bad value for some $j^{\prime}<j$.

We define a $j$-bad tree rooted at a variable $v$ as follows:

- A 0-bad tree rooted at $v$ is a set of $b_{0}$ hyperedges intersecting at $v$ such that for each 0 -bad value $\delta$, one of the hyperedges receives the constraint $C(\delta)$ with $X_{i(\delta)}$ mapped onto $v$.

- For $j \geq 1$, a $j$-bad tree rooted at $v$ consists of a $j^{\prime}$-bad tree $T_{j^{\prime}}$ rooted at $v$ for each $0 \leq j^{\prime}<j$, along with $b_{j}$ hyperedges $e_{1}, \ldots, e_{b_{j}}$ intersecting at $v$ such that:

(i) for each $j$-bad value $\delta$, a hyperedge $e_{i}$ receives the constraint $C(\delta)$ with $X_{i(\delta)}$ mapped onto $v$; and

(ii) for every value $u \neq v$ in one of the $e_{i}, u$ is the root of a $(j-1)$-bad tree $T_{u}$; and

(iii) all of the bad trees $\left\{T_{0}, \ldots, T_{j-1}\right\} \cup\left\{T_{u}: u\right.$ is in some $\left.e_{i}\right\}$ are disjoint except that $T_{0}, \ldots, T_{j-1}$ all contain $v$. 
It is not hard to argue inductively that if $v$ roots a $j$-bad tree then $v$ cannot recieve a $j^{\prime}$-bad value for any $j^{\prime} \leq j$ : If $j=0$ then for each 0 -bad value $\delta$, $v$ is forbidden $\delta$ by $C(\delta)$. For $j \geq 1$, since for each $j^{\prime}<j$, $v$ roots a $j^{\prime}$-bad tree, $v$ cannot receive a $j^{\prime}$-bad value. if $v$ recieves some $j$-bad value, $\delta$, then since $v$ lies in a $C(\delta)$-constraint, at least one of the other variables in that constraint must receive a $j^{\prime}$-bad value for some $j<j$. But each of those variables roots a $j^{\prime}$-bad tree and thus cannot take any such bad value.

A bad tree is a $j$-bad tree where $j$ is the maximum value such that $b_{j}>0$. If 1 ' does not hold, then every value is a $j^{\prime}$-bad value for some $j^{\prime}<j$ and so the root of a bad tree cannot receive any of them. Therefore, if a CSP contains a bad tree then it is unsatisfiable.

Now, we show that for any distribution $\mathcal{P}$ and $c>0$, a.s. $C S P(\mathcal{P})$ contains a bad tree. The following fact is well known in random graph theory.

Fact: For any fixed hypertree $T$ independent of $n$, there exists a constant $z>0$ such that a.s. our random constraint hypergraph will contain at least $\zeta n$ copies of $T$.

The basic outline for the proof of this fact is as follows: (1) The expected number of copies of $T$ is $\zeta^{\prime} n$ by a simple but tedious calculation. (2) Because $n$ grows much larger than the (constant) size of $T$, individual potential appearances of $T$ occur nearly independently. An application of Chebychev's inequality formalizes this and shows that the number of copies of $T$ is highly concentrated. In particular, it is a.s. at least $\zeta n$ for any $\zeta<\zeta^{\prime}$. See [7] for details of the case $k=2$. The analysis for higher $k$ is the same.

Naturally, we will apply this fact where $T$ is equal to the underlying hypertree of a minimum sized bad tree. Let $A_{1}$ be the event that our hypergraph contains at least $\zeta n$ copies of $T$. If $A_{1}$ holds then using a simple greedy procedure as in the proof of Theorem 1, we can easily find a set $I$ of $\frac{\zeta}{|T|} n$ disjoint copies of $T$ (where $|T|$ denotes the number of vertices in $T$ ). Upon choosing the constraints, a copy becomes bad with probability $p_{1}$ for some $p_{1}>0$ which is not a function of $n$. Therefore, the probability that our CSP contains no bad trees is at most

$$
\operatorname{Pr}\left(A_{1}\right)+\left(1-p_{1}\right)^{\frac{\zeta}{|T|} n}=o(1)
$$

To prove that if 1 ' holds then for any $\mathcal{P}$ and $c<\frac{1}{k(k-1)}, C S P(\mathcal{P})$ is not a.s. unsatisfiable we just have to show that any CSP with constraints from $\mathcal{C}$ and whose constraint hypergraph is a tree is satisfiable.

For such a CSP, choose any variable $v$ to be the root. Assign $v$ a good value. Since $v$ is good, it is possible to assign good values to every variable which shares a constraint with $v$ without violating any of those constraints. Now continue through the CSP as in the proof of Theorem 1, each time assigning only good 
values, thus satisfying the CSP.

The remainder of the proof, namely the relevance of property 2 , is identical to the proof of Theorem 1.

Proof of Theorem 5: This follows almost exactly like the proof of Theorem 2 .

If property 3' does not hold, then with probability tending to some positive constant $C S P(\mathcal{P})$ will contain a subproblem whose constraint hypergraph is a unicyclic component and (i) the cycle of that component is constrained such that at least one variable must receive a bad value, and (ii) each variable on that cycle roots a bad tree. Clearly, such a subproblem is unsatisfiable.

If property 3 ' holds, then it is enough to show that any CSP with constraints from $\mathcal{C}$ and whose constraint hypergraph is unicyclic is satisfiable. To satisfy such a CSP, first assign good values to each variable on the cycle without violating any of the cycle constraints, and then continue to assign good values to each of the other variables as in the proof of Theorem 4.

Theorem 3 also applies to the asymmetric case. Again, it would be interesting to characterize the distributions $\mathcal{P}$ for which $C S P(\mathcal{P})$ is a.s. unsatisfiable with $M=c n$ for all $c>\frac{1}{k(k-1)}$.

\section{Sharp thresholds}

If a model $\operatorname{CSP}(\mathcal{P})$ exhibits a transition, then it is natural to ask if it satisfies the following stronger property:

There exists a constant $c>0$ such that for any $\epsilon>0$, if $M<(c-\epsilon) n$ then $\operatorname{CSP}(\mathcal{P})$ is a.s. satisfiable and if $M>(c+\epsilon) n$ then $\operatorname{CSP}(\mathcal{P})$ is a.s. satisfiable.

The current "state-of-the-art" in the analysis of sharp thresholds does not allow us to prove a property this strong even for CNF-satisfiability. Instead, we must weaken the property slightly as follows.

$\operatorname{CSP}(\mathcal{P})$ is said to have a sharp threshold of satisfiabilty if there exists a function $c(n)$ bounded away from 0 such that for any $\epsilon>0$, if $M<(c(n)-\epsilon) n$ then $C S P_{n, M}(\mathcal{P})$ is a.s. satisfiable and if $M>(c(n)+\epsilon) n$ then $C S P_{n, M}(\mathcal{P})$ is a.s. satisfiable.

Note that the only difference between this and the stronger property is that here we allow $c$ to vary with $n$. All natural models which are known to have sharp thresholds are conjectured to also satisfy the stronger property where $c(n)=c$ for all $n$. However, we do not know how to prove this. 
This notion of sharp thresholds was introduced (in a broader context than that in the preceding definition) by Friedgut[10] who proved that random instances of $k$-SAT exhibit a sharp threshold. In doing so, he proved a general theorem characterizing random graph properties which have sharp thresholds. To describe this theorem, we must introduce some definitions.

A graph property, $P$, is monotonically increasing if (i) $P$ is invarient under graph automorphisms, and (ii) whenever $H$ is a subgraph of $G$, and $H$ has $P$, then $G$ must have $P$. This definition extends in the obvious manner to properties of CSP's - condition (ii) becomes: if $F_{1}, F_{2}$ are CSP's where every contraint in $F_{1}$ is also contained in $F_{2}$, and if $F_{1}$ has $P$ then $F_{2}$ must have $P$. The only property which concerns us in this paper is that of being unsatisfiable, which is clearly monotonically increasing.

Given two properties $P_{1}, P_{2}$, their symmetric difference, $P_{1} \triangle P_{2}$ is the property of satisfying one but not both of $P_{1}, P_{2}$. Given a set of graphs $\mathcal{H}=$ $\left\{H_{1}, \ldots, H_{k}\right\}$, we define $Q(\mathcal{H})$ to be the property of having a subgraph which is isomorphic to one of the members of $\mathcal{H}$.

$G_{n, M}$ is the random graph with $n$ vertices and $M$ edges, where each such graph is equally likely to be chosen.

We extend our definitions of transition and sharp theshold to the setting of random graphs in the obvious manner. A monotonically increasing graph property, $P$, exhibits a transition if there exists $c_{1}, c_{2}>0$ such that if $M<c_{1} n$ then a.s. $G_{n, M}$ does not have $P$, and if $M>c_{2} n$ then a.s. $G_{n, M}$ has $P$. A graph property $P$ with a transition has a sharp threshold if there exists a function $c(n)$ bounded away from 0 such that for any $\epsilon>0$, if $M<(c(n)-\epsilon) n$ then a.s. $G_{n, M}$ does not have $P$, and if $M>(c(n)+\epsilon) n$ then a.s. $G_{n, M}$ has $P$.

Friedgut's Theorem yields the following:

Friedgut's Theorem[10] $]^{6}$ If a monotonically increasing graph property $P$ with a transition does not have a sharp threshold then there exists some $c(n)$ such that for every $\delta>0$ there is a fixed set of unicyclic graphs $\mathcal{H}=\left\{H_{1}, \ldots, H_{k}\right\}$ (this set depends only on $\delta$ and not $n$ ) such that, when $M=c(n) \times n$, the probability of $G_{n, M}$ having $P \triangle Q(\mathcal{H})$ is less than $\delta$.

In other words, when $M=c(n), P$ can be arbitrarily closely approximated in probability by the property of having a subgraph from a list of unicyclic graphs.

This theorem also extends to random hypergraphs and to random CNF-

\footnotetext{
${ }^{6}$ Friedgut's Theorem is in fact much more general than this, in that (i) it shows that this is essentially an "if and only if" statement, and (ii) it allows for the possibility of a sharp threshold which occurs when $M$ is not linear in $n$. We only include this version here for the sake of simplicity, and because it covers all situations which are relevant to this paper.
} 
formulae. It is natural to expect that it might extend to the random CSPmodels discussed here, i.e. that $C S P(\mathcal{P})$ has a sharp threshold of satisfiability iff unsatisfiability cannot be arbitrarily well approximated by containing a subproblem isomorphic to one of a list of problems whose constraint hypergraphs are unicyclic. If $C S P(\mathcal{P})$ exhibits a transition then all problems with unicyclic constraint hypergraphs whose constraints are drawn from $\mathcal{C}$ are satisfiable. Thus, it would be natural to further expect that $C S P(\mathcal{P})$ has a sharp threshold of satisfiability iff it exhibits a transition. However, this is not the case, as the following example shows:

Example 1: Suppose that $k=2, d=4$, and that $\mathcal{C}$ contains exactly two constraints. The first one, $C_{1}$, forbids $\left(X_{1}, X_{2}\right)$ from receiving any pair of values from $\{(1,1),(2,2),(3,3),(4,4)\}$. The second, $C_{2}$, forbids any pair $(x, y)$ where one member of the pair is from $\{1,2\}$ and the other member is from $\{3,4\}$. $\mathcal{P}\left(C_{1}\right)=\frac{1}{3}$ and $\mathcal{P}\left(C_{2}\right)=\frac{2}{3}$.

Note that it is easy to verify that $C S P(\mathcal{P})$ exhibits a transition.

Suppose that $M=c n$ where $\frac{3}{4}<c<\frac{3}{2}$. Let $G_{i}$ be the constraint graph induced by constraints of type $C_{i}$. A.s. $G_{1}$ will have $\frac{1}{3} M+o(M)=\left(\frac{1}{2}-c_{1}\right) n+$ $o(n)$ edges and $G_{2}$ will have $\left(\frac{1}{2}+c_{2}\right) n+o(n)$ edges for some constants $c_{1}, c_{2}>0$. Therefore, by the most fundamental theorem in random graph theory (see [9]), a.s. no component of $G_{1}$ will have size greater than $O(\log n)$, while $G_{2}$ will have a giant component of size $\alpha n+o(n)$ for a constant $\alpha=\alpha(c)>0$, and every other component of $G_{2}$ will have size at most $O(\log n)$.

Let $T$ be the vertices of the giant component of $G_{2}$. Define $B$ to be the event that $G_{1}$ has an odd cycle whose vertices all lie in $T$. It is easy to see that if $B$ holds then our CSP is unsatisfiable: the $C_{2}$ constraints imply that either all variables in $T$ recieve 1 or 2 , or they all receive 3 or 4 . However, if a set of vertices belongs to an odd cycle in $G_{1}$ then amongst them there must be at least 3 values. Furthermore, it is not difficult to verify that the probability that $B$ does not hold AND the CSP is unsatisfiable is o(1). Therefore, the probability of satisfiability is $1-\operatorname{Pr}(B)+o(1)$. It is also easily computed that $\operatorname{Pr}(B)$ tends to a constant (in terms of $c$ ) which is strictly between 0 and 1 , and so our CNF is neither a.s satisfiable nor a.s. unsatisfiable - $G_{1}$ and $G_{2}$ are very close to being two independent random graphs, so this is esssentially the probability that a random graph with $\alpha n$ vertices and $\left(\frac{1}{2}-c_{1}\right) n$ edges contains an odd cycle. Therefore this model does not have a sharp threshold despite the fact that it exhibits a transition.

Dimitris Achlioptas[2] pointed out that for this particular example, there is a set of problems of the type which Friedgut's Theorem guarantees for random graphs and instances of $k$-SAT when a property has a coarse threshold. I.e., there is a set of problems such that having a subproblem from this set is a good approximation of being unsatisfiable. For each $i$, let $\mathcal{H}_{i}$ denote the set of 
subproblems such that, when $G_{1}, G_{2}$ are defined as above,

(i) $G_{1}$ is a cycle of length $2 r+1$ for some positive integer $r$, with vertices $v_{1}, \ldots, v_{2 r+1}$

(ii) $G_{2}$ consists of $r$ disjoint trees $T_{1}, \ldots, T_{r}$ each of size $i$;

(iii) for each $i, v_{i}$ is in $T_{i}$, and these are the only vertices that $G_{1}, G_{2}$ have in common.

Let $P$ be the property that our random problem is unsatisfiable. For any $\delta>0$, we can choose $i$ large enough that $\operatorname{Pr}(P \triangle Q(\mathcal{H}))<\delta$. Almost surely, if $G_{1}$ has an odd cycle lying in the giant component of $G_{2}$ then breadthfirst searchs, in $G_{2}$, from each vertex of the cycle will yield a collection of disjoint trees of length $i$, for any constant $i$. Therefore, $\operatorname{Pr}\left(P-Q\left(\mathcal{H}_{i}\right)\right)=o(1)$. On the other hand, the probability that $G_{1}$ has a cycle through the vertex of any non-giant component of $G_{2}$ which has size at least $i$, tends to a constant $\delta_{i}$ which tends to 0 as $i$ grows. Choosing $i$ so that $\delta_{i}<\delta$ yields $\operatorname{Pr}\left(Q\left(\mathcal{H}_{i}\right)\right)<\delta$. We omit the details, which are straightforward to a reader who is experienced in random graph theory.

It would be quite interesting to determine some neccessary and sufficient conditions on $\mathcal{P}$ for $C S P(\mathcal{P})$ to have a sharp threshold. We close this section by noting the following sufficient condition:

\section{Theorem 6 If}

(a) $\mathcal{C}$ is very well-behaved;

(b) every constraint in $\mathcal{C}$ has the property that for each value $\delta$ and canonical variable $X_{i}$, there is at most one restriction with $X_{i}=\delta$; and

(c) for each $C \in \mathcal{C}, \mathcal{P}(C)=\frac{1}{|\mathcal{C}|}$;

then $C S P(\mathcal{P})$ has a sharp threshold.

Remark 3: It is not difficult to weaken conditions (b) and (c) somewhat, but (a) is, of course, neccessary.

Note that this theorem generalizes the fact that random instances of $k$-SAT have a sharp threshold for $k \geq 3$ and the fact that $k$-colourability of random graphs has a sharp threshold for $k \geq 3$. This latter fact was proven by Achlioptas and Friedgut[3], and the proof of Theorem 6 is nearly identical to their proof. To avoid a long repetition of a proof which is readily available, we only give an outline of our proof. 
Proof of Theorem 6: Rather than using Friedgut's Theorem, we we will make use of a similar theorem proved by Bourgain, subsequently to Friedgut's proof. The statement of this theorem is somewhat weaker, but it is easily applied to a more general situation, including the random models considered here.

Consider a set $\mathcal{A}$ of items $\left\{A_{1}, \ldots, A_{r}\right\}$, and a selection probability $p$. We will choose a random subset $A \subseteq \mathcal{A}$ as follows: for each $A_{i}$, we make an independent choice as to whether to include $A_{i}$ in $A$, placing it in $A$ with probability $p$. For example, if $\mathcal{A}$ is the set of possible edges of an $n$ vertex graph, then we are simply choosing the random graph $G_{n, p}$, which is well known (see, for example, [7]) to be in many ways equivalent to $G_{n, M}$ where $M=p\left(\begin{array}{l}n \\ 2\end{array}\right)$. Under the assumption that $\operatorname{Pr}(C)$ is rational for each $C \in \mathcal{C}$, we can also simulate $C S P(\mathcal{P})$ using this model as follows:

Following Remark 2, we will work in the equivalent model whereby we select each possible hyperedge to be in the constraint hypergraph independently with probability $p=\frac{c \times k !}{n^{k-1}}$.

$\mathcal{A}$ is the set of all $\left(\begin{array}{l}n \\ k\end{array}\right) k !|\mathcal{C}|$ possible constraints. We place each constraint into $A$ with selection probability $p^{\prime}$ (to be named later), and we let our CSP consist of each constraint $C$ such that (i) $C \in A$, and (ii) none of the other $k !|\mathcal{C}|-1$ possible constraints on the same $k$-tuple of variables is in $A$. For any $k$-tuple of variables, that $k$-tuple forms a constraint with probability exactly $k !|\mathcal{C}| \times p^{\prime}\left(1-p^{\prime}\right)^{k !|\mathcal{C}|-1}$, and we choose $p^{\prime}$ so that this value is equal to $p$. Furthermore, each member of $\mathcal{C}$ is equaly likely to be that constraint. Thus, this represents our model precisely. (Note that $p^{\prime}$ is very close to $p$ - in fact $p^{\prime}=p\left(1+O\left(n^{-(k-1)}\right)\right.$.)

This transformation allows us to apply Bourgain's Theorem to $C S P(\mathcal{P})$. As with Friedgut's Theorem, we will not state Bourgain's Theorem in it's full power - instead, we will only state it's implications to this setting, thus avoiding some technicalities.

Bourgain's Theorem [10] Suppose that for each $c \in \mathcal{C}, \mathcal{P}(C)=\frac{1}{|\mathcal{C}|}$. If $C S P(\mathcal{P})$ does not have a sharp threshold of satisfiability then there exists $c(n)$ and absolute constants $T, 0<\gamma<1$ and $\beta>0$ such that when $M=c(n) \times n$, the probability that $C S P(\mathcal{P})$ is satisfiable is $\gamma+o(1)$ and either

(i) with probability at least $\beta, C S P(\mathcal{P})$ contains an unsatisfiable subproblem $A_{1}$ of size at most $T$; or

(ii) there exists a satisfiable subproblem $A_{1}$ of size at most $T$ such that conditioning on $C S P(\mathcal{P})$ containing $A_{1}$ lowers the probability of satisfiability to below $\gamma-\beta$.

A straightforward and well known ${ }^{7}$ random graph argument implies that

\footnotetext{
${ }^{7}$ See $[7]$ for a proof of the case $k=2$. The proof for higher values of $k$ is similar.
} 
a.s. every subproblem with at most $T$ constraints is unicyclic. If $\mathcal{C}$ is very well behaved, all such problems are satisfiable. Thus, (i) does not hold and so we only need to eliminate the possibility of (ii).

So consider a CSP $A_{1}$ as in (ii). Without loss of generality, we can assume that $A_{1}$ is on the variables $x_{1}, \ldots, x_{t}$ for some $t \leq T$. Consider any satisfying assignment $x_{1}=\delta_{1}, \ldots, x_{t}=\delta_{t}$ for $A_{1}$. The probability that $C S P(\mathcal{P})$ is satisfiable, conditional on $C S P(\mathcal{P})$ containing $A_{1}$ is at most the probability that $C S P(\mathcal{P})$ has a satisfying assignment with $x_{1}=\delta_{1}, \ldots, x_{t}=\delta_{t}$.

Let $\mathcal{D}$ be the set of constraints, other than those in $A_{1}$, which involve $x_{1}, \ldots x_{t}$. A straightforward argument shows that there is an absolute constant $R$ such that with probability at least $\frac{1-\beta}{2}$ (a) $|\mathcal{D}| \leq R$, (b) no constraint in $\mathcal{D}$ involves two variables from $\left\{x_{1}, \ldots, x_{t}\right\}$ and (c) no variable outside of $\left\{x_{1}, \ldots, x_{t}\right\}$ lies in more than one constraint in $\mathcal{D}$.

Consider any constraint $D \in \mathcal{D}$, say on variables $x_{i}, x_{j_{1}}, \ldots, x_{j_{k-1}}$ where $1 \leq i \leq t$ and each other index is greater than $t$. Since each constraint in $\mathcal{C}$ contains at most one restriction involving $x_{i}=\delta_{i}$, insisting that $x_{i}=\delta_{i}$ at worst reduces to imposing a single restricition of size $k-1$ on $x_{j_{1}}, \ldots, x_{j_{k-1}}$. This is no more restrictive than forbidding a single value for each $x_{j_{l}}$. Thus, conditional on (a), (b) and (c) holding, the probability that $C S P(\mathcal{P})$ has a satisfying assignment with $x_{1}=\delta_{1}, \ldots, x_{t}=\delta_{t}$, is bounded from below by the following experiment:

Fix some collection of values $\zeta_{1}, \ldots, \zeta_{R \times(k-1)}$. Choose a random CSP from $C S P(\mathcal{P})$ with $M=c(n)$. Pick $R \times(k-1)$ variables at random, and add the additional requirements that the $i$ th variable cannot recieve $\zeta_{i}$.

Since the probability that either (a), (b) or (c) fails is at most $\frac{\beta}{2}$, condition (ii) implies that these additional requirements do not decrease the probability of satisfiability by more than $\frac{\beta}{2}$. We will show that this is impossible. To do so requires two steps:

(1) We show that if adding these $R \times(k-1)$ additional requirements decreases the probability of satisfiability by at least $\frac{\beta}{2}$, then so would increasing $M$ from $c(n) \times n$ to $c(n) \times n+R^{\prime}$, for an absolute constant $R^{\prime}$ defined in terms of $R \times(k-1)$.

(2) Increasing $M$ from $c(n) \times n$ to $c(n) \times n+R^{\prime}$ will only decrease the probability of unsatisfiability by $o(1)$.

Step (2) is at least intuitively obvious - adding a relatively very small number of constraints should not have a significant effect on the probability of satisfiability. However, it takes a little work to prove it.

The proof of Step (1) goes as follows: Suppose that we were only adding one additional requirement - we pick a random variable and forbid it from recieving 
$\zeta_{1}$. Let $X_{1}$ be the set of variables such that, before this requirement, the only satisfying assignments set all of $X_{1}$ equal to $\zeta_{1}$. If this requirement causes our problem to turn from satisfiable to unsatisfiable then we must have chosen a random variable in $X_{1}$. Thus, in order for this to have a significant probability of happenning, $X_{1}$ would have to contain at least $\alpha n$ variables for some constant $\alpha>0$ - suppose that this is the case. Then if instead of adding this extra requirement, we added a single additional constraint, we would cause the CSP to become unsatisfiable with probability at least $\alpha^{k} \times \frac{1}{\text { calc }}$ which is a positive constant. The reason is that this is the probability that the entire $k$-tuple is from $X_{1}$ and that we choose the restriction which forbids them from all being assigned the value $\zeta_{1}$. Thus adding an additional constraint has a serious affect on the probability of satisfiability.

Modifying this argument to the case where we have $R(k-1)$ additional requirements uses the same ideas, but is is technically a little complicated.

The proofs of Steps (1) and (2) are identical to the corresponding steps found in [3], [10] and [1]. We refer the reader to either of those for more details.

\section{Generating Instances}

In practical terms, generating random CSP's from $C S P(\mathcal{P})$ can sometimes be awkward. In this section, we will discuss how to do this fairly simply for some distributions $\mathcal{P}$ with transitions and partial transitions. We will focus on symmetric $\mathcal{C}$, since they are a little simpler to deal with.

The most easily dealt with model with a partial transition is probably the case where $\mathcal{C}$ contains all constraints which do not forbid any values. Rather than list the members of $\mathcal{C}$ explicitly, it is probably simpler to do the following: Choose $M$ random constraints, one-at-a-time. Each time, first select the $k$ tuple of variables and then select a random constraint using any distribution you please, without putting any effort into ruling out constraints which forbid values. After selecting your constraint, run a quick check to see if it forbids any values - if it does, then reject it and pick another constraint. Repeat until you have chosen a constraint in $\mathcal{C}$. As long as your distribution does not make it overwhelmingly likely that your constraint will forbid a value, you will not expect to have to reject very many constraints.

Choosing from a distribution with a transition is much more awkward, since it is not always easy to tell quickly whether a set $\mathcal{C}$ can form an unsatisfiable cyclic CSP, let alone sample easily from such a set using rejection. In [11], Gent et. al. present a particular distribution which exhibits a transition and which is reasonably easy to sample from. An alternate approach is as follows. 
The main problem with sets $\mathcal{C}$ which have partial transitions but not transitions is that with positive probability they will form unicyclic components which are unsatisfiable. To avoid this problem, one can choose a CSP using a $\mathcal{C}$ with a partial transition as described above, and then run a quick check looking for unsatisfiable unicyclic components. If any are found, then delete them from the CSP. With very high probability, you will not delete very many constraints, and so you will still be left with a significant CSP. With a little more work, you can search for and remove any "small" unicyclic unsatisfiable subproblems, even those which do not form components.

Strictly speaking, this does not choose a CSP from $\operatorname{CSP}(\mathcal{P})$ for any distribution $\mathcal{P}$, but for experimental purposes, it will often be very useful for generating some large difficult CSP's.

\section{References}

[1] D. Achlioptas. Threshold Phenomena in Random Graph Colouring and Satisfiability. Ph.D. Thesis, Dept. of Computer Science, University of Toronto, 1999.

[2] D. Achlioptas. Personal communication.

[3] D. Aclioptas and E. Friedgut. A threshold for $k$-colourability. Random Structures and Algorithms 14 (1999), 63 - 70.

[4] D. Achlioptas, L. Kirousis, E. Kranakis, D. Krizanc. Rigorous results for random $(2+p)$-SAT. Proceedings of RALCOM '97 (1997), $14-23$.

[5] D. Achlioptas, L. Kirousis, E. Kranakis, D. Krizanc, M. Molloy, and Y. Stamatiou. Random constraint satisfaction: a more accurate picture. (submitted).

[6] N. Alon and J. Spencer, The Probabilistic Method. Wiley (1992).

[7] B. Bollobas. Random Graphs. Academic Press (1985).

[8] P. Cheeseman, B. Kanefsky and W.M. Taylor. Where the really hard problems are. Proceedings of the 12th IJCAI (1991), $331-337$.

[9] P. Erdos and A. Renyi On the evolution of random graphs. Publication of the Mathematical Institute of the Hungarian Academy of Sciences, 5 (1960), 17-61.

[10] E. Friedgut and an appendix by J. Bourgain. Sharp thresholds of graph properties and the $k$-SAT problem. J. Am. Math. Soc. 12 (1999), 1017 1054. 
[11] I. Gent, E. MacIntyre, P. Prosser, B. Smith and T. Walsh. Random constraint satisfaction: flaws and structure. (submitted).

[12] R. Karp. The transitive closure of a random digraph. Random Structures and Algorithms 1 (1990), 73 - 93. 